\title{
Contrato psicológico y compromiso organizacional: un estudio bibliométrico
}

\author{
Juan Herrera Ballesteros \\ Universidad de Málaga \\ juanherrera@uma.es \\ Carlos J. de las Heras Rosas \\ Universidad de Málaga \\ chr@uma.es
}

Resumen: La literatura encargada de estudiar el contexto laboral ba llevado a cabo multitud de trabajos relacionados con los conceptos "contrato psicológico " $y$ "compromiso organizacional" de forma separada. Se han demostrado el nivel de relación causal que tienen ambos conceptos y su importancia sobre la eficiencia de las organizaciones empresariales. El objetivo de este trabajo es conocer cuál es la producción científica existente, profundizar en los enfoques que predominan y cuáles son los que aún quedan por explorar. Para ello, se ha optado por utilizar como técnica el estudio bibliométrico. Se han analizado detalladamente un total de 372 artículos procedentes de revistas catalogadas en el Social Science Citation Index (SSCI), Science Citation Index Expanded (SCI-EXPANDED) y Scopus. La aplicación de técnicas bibliométricas ha permitido analizar y presentar la evolución de las publicaciones de este ámbito mediante indicadores de actividad y de relación. Los resultados obtenidos sugieren que el interés por el estudio sobre el contrato psicológico y el compromiso organizacional se mantiene vigente, aunque no se prevé un incremento importarte. Se ba podido comprobar que la literatura ha abordado las cuestiones más importantes sobre el contrato psicológico y compromiso organizacional en las organizaciones empresariales, sin embargo, temas tan sensibles como la gestión de la diversidad, la repercusión en 
la igualdad de género o aspectos sobre la cultura organizacional son prácticamente testimoniales.

Palabras clave: organizaciones empresariales; gestión de RR. HH.; factores motivacionales; eficiencia organizacional, diversidad.

Abstract: The literature on employment contains a multitude of works related to the concepts of psychological contract and organizational commitment. The level of causal relationship that both concepts have has been demonstrated, as has their importance regarding the efficiency of business organizations. The objective of this study is to examine the existing scientific literature, to study in depth the predominant approaches and to indentify which ones remain to be explored. This has been achieved by a bibliometric study. A total of 372 articles from journals catalogued in the Social Science Citation Index (SSCI), Science Citation Index Expanded (SCI-EXPANDED) and Scopus have been analyzed in detail. The application of bibliometric techniques has made it possible to analyze and present the evolution of publications in this field, by means of activity and relationship indicators. The results obtained suggest that the psychological contract and organizational commitment remain topics of interest, although no significant increase is expected. The study has verified that the literature has addressed the most important questions regarding the psychological contract and organizational commitment in business organizations; however, sensitive issues such as diversity management, impact on gender equality or aspects of organizational culture have received little attention.

Keywords: business organizations; HR management; motivational factors; organizational efficiency; diversity. 


\section{Introducción}

En el contexto empresarial, se requiere un cambio hacia un modelo de eficiencia basado en el compromiso organizacional. Las organizaciones empresariales necesitan formar equipos altamente comprometidos con sus objetivos estratégicos, solo en un entorno en el que la relación entre empresa y trabajador sea equilibrada se pueden mantener altas cuotas de compromiso organizacional. La literatura en multitud de trabajos ha relacionado el compromiso organizacional con el cumplimiento del contrato psicológico, es decir, con el grado del cumplimiento de las promesas realizadas por parte de la organización (Rousseau y Parks, 1993), enmarcándolo como variable explicativa y determinante del compromiso organizacional (Zaragoza, 2013; Guest, 1998).

El objetivo de este trabajo es conocer a través de la producción científica publicada cómo se entroncan los conceptos "contrato psicológico" y "compromiso organizacional". Para ello, se utilizarán técnicas bibliométricas de análisis que permiten presentar un detallado estudio sobre la evolución de los artículos y autores que han abordado esta temática. Los enfoques adoptados y la incorporación de numerosos estudios empíricos han proporcionado una notable riqueza literaria sostenida, que va desde los primeros trabajos publicados en la década de los noventa del pasado siglo hasta la proliferación de publicaciones que acontecen en los inicios del siglo xxI. La aportación de este estudio estriba en dar visibilidad a resultados científicos que servirán para las organizaciones empresariales, como instrumentos en la toma de decisiones en su gestión laboral, y para la comunidad científica, como conocimiento de los espacios de investigación por explorar sobre contrato psicológico y compromiso organizacional.

No existe una categorización generalmente aceptada que englobe las temáticas tratadas sobre la investigación de la conexión de estos dos constructos teóricos. En este artículo el lector encontrará, en primer lugar, una breve contextualización y muestra científica de los dos conceptos que nos ocupan: contrato psicológico y compromiso organizacional. La evolución de ambos conceptos por separado y su conexión desde múltiples enfoques ha generado un amplio espectro de investigaciones que es preciso analizar. En este punto, se citan los dos tipos aceptados de contrato psicológico, el transaccional y el relacional. Asimismo, se detallan las tres perspectivas del compromiso organizacional: intercambio social; perspectiva psicológica y perspectiva de atribución.

Las fuentes empleadas en la búsqueda de publicaciones científicas han sido Web of Science (WOS) y Scopus. Se ha partido de un total de 372 artículos relacionados con la temática que nos ocupa. Un posterior análisis de los resultados ha seleccionado un total de 207 trabajos que forman el punto de partida del aná- 
lisis bibliométrico que se realiza a continuación. Estas dos centenas de artículos han sido publicados en 86 revistas distintas, para un total de 28 países y más de 300 autores. Las investigaciones examinadas van desde "The institutionalization of organizational ethics", publicado por Sims en Journal of Business Ethic en 1991, hasta "Measuring the impact of knowledge loss: a longitudinal study", de Massinghan, en 2018 en Journal of Knowledge Management.

El análisis efectuado se divide en dos etapas: una primera relacionada con la actividad y producción científica desde una orientación cuantitativa y una segunda donde la relación y enfoque utilizados por autores e instituciones centran el cuerpo de la investigación. Las técnicas cuantitativas y los cruces de copalabras y coocurrencias han permitido exponer a modo de panel cuáles han sido los países, autores y revistas más prolíficos; asimismo, han mostrado la tendencia temática en este campo y su evolución a lo largo del tiempo. Las investigaciones indican que los conceptos "contrato psicológico" y "compromiso organizacional" se han estudiado tanto de forma independiente como a través de su relación. Se han encontrado diferentes enfoques o perspectivas que han estudiado contrato psicológico y compromiso organizacional. Determinados trabajos han abordado ambos conceptos como eje principal, sin embargo, hay otros que lo han hecho de forma separada y utilizando moderadores, otras investigaciones han estado relacionadas con aspectos organizacionales, con la gestión de RR. HH., gestión de la diversidad o con factores motivacionales.

\section{Contrato psicológico y compromiso organizacional}

El contrato psicológico es uno de los conceptos clave en la relación entre trabajador y empresa. Argyris (1960) es el primer autor que lo define, describiéndolo como "las percepciones de ambas partes de la relación laboral, organización e individuo, de las obligaciones implícitas en la relación. El contrato psicológico es el proceso social por donde se llega a estas percepciones". Schein (1982) define los contratos psicológicos como un conjunto de expectativas recíprocas entre empleado y organización, a su vez puntualiza que se trata de un determinante en el comportamiento.

El contrato psicológico se supone clave en la relación, ya que se basa en las percepciones del trabajador como consecuencia de su relación laboral, concretamente con el grado y cumplimiento de las promesas realizadas por parte de la organización (Rousseau y Parks, 1993), afectando directamente sobre el compromiso organizacional (Guest, 1998), la satisfacción individual, y, a su vez, el resultado del desempeño. Para Rousseau (1995) un contrato psicológico es un modelo mental particular que permite al trabajador enmarcar aspectos relacio- 
nados con su ambiente organizacional, tales como las promesas realizadas, las condiciones del empleo y la confianza establecida. Así pues, las expectativas son el punto de referencia con el que los trabajadores comparan los resultados obtenidos en su relación laboral. Además, los resultados de esta comparación conllevan una serie de reajustes en el comportamiento que implican adaptar o equilibrar las diferencias percibidas.

Desde el punto de vista del tipo de relación laboral se conciben diferencias de apreciación sobre cómo pueden afectar el incumplimiento de expectativas o el incumplimiento del contrato psicológico. En este sentido, aquellos trabajadores que tienen un contrato temporal pueden entender su relación con la empresa de una manera más superficial y, por lo tanto, pueden percibir de diferente forma el incumplimiento de promesas, e incluso puede que no lo vean como tal, sin embargo, aquellos trabajadores que tienen un mayor arraigo debido a un tipo de contrato indefinido o mayor antigüedad en la empresa podrían ser más sensibles y esperar con más firmeza que se cumplan sus expectativas. En esta línea, Rousseau (1995) y Arnold (1996) plantean que existen dos tipos de contrato psicológico, el contrato transaccional y el contrato relacional. El primero está más centrado en las recompensas materiales, tratándose normalmente de empleados con sueldos muy competitivos, con poca o nula lealtad hacia la empresa, con bajo sentimiento de pertenencia y con perspectiva de transitoriedad. Esta conceptualización no está relacionada solo con trabajadores con contratos temporales, sino que se la relaciona también con trabajadores que, aunque tengan un contrato indefinido, mantienen un desarraigo similar, conllevando una baja posibilidad de asumir responsabilidades de medio o largo plazo. En el caso del segundo, el contrato relacional está más centrado en lo intangible, en aspectos relacionales, de crecimiento personal, de sentimiento de pertenencia, y existe, por tanto, un grado mayor de lealtad por parte de los empleados, y la empresa lo percibe como la obligación de proporcionar seguridad a sus trabajadores. El contrato psicológico, por tanto, podría estar formado en un continuo entre el contrato transaccional, basado exclusivamente en intercambios económicos, y el relacional, más orientado a aspectos socioemocionales, como, por ejemplo, lealtad (Tena, 2002).

En cuanto a la conceptualización del compromiso organizacional, la literatura arroja tres perspectivas que tratan de explicar este constructo. La primera, desde un punto de vista del intercambio social, pone énfasis en el compromiso que el individuo alcanza con la organización fruto de las pequeñas inversiones que este ha realizado a lo largo del tiempo, y que continúa en esta para no sacrificarlas (Becker, 1960). La segunda, desde una perspectiva psicológica, pone el foco en la fuerza vinculante entre persona y organización, y se caracteriza por el deseo de permanecer como miembro manteniendo un alto nivel de esfuerzo por la orga- 
nización, y aceptar los valores y metas de esta, a cambio de recibir determinadas recompensas psicológicas, tales como apoyo o reconocimiento (Mowday, Steers y Porter, 1979; O’Reilly y Chatman, 1986; Mathieu y Zajac, 1990). La tercera tiene que ver con la perspectiva de atribución, que está más relacionada con la ética del trabajo y con la responsabilidad que el trabajador adquiere, y que le impulsa a hacer un esfuerzo por realizar bien su trabajo (Varona, 1993). En cuanto a las definiciones más aceptadas se encuentra la ofrecida por Porter y Lawer (1965), quienes le dan un enfoque del compromiso orientado a la vinculación hacia la empresa por parte del trabajador, y lo definen como el deseo de realizar elevados esfuerzos por el bien de la institución, el anhelo de permanecer en esta y aceptar sus principales objetivos y valores; sin embargo, Cotton (1993) lo focaliza de manera más procedimental, englobándolo en un proceso participativo, resultado de combinar información, influencia y/o incentivos, donde se usan todas las capacidades personales para estimular el apego de los empleados hacia el éxito institucional. En definitiva, la formación del compromiso organizacional está relacionada con los inputs que el trabajador recibe de la organización e íntimamente ligada a los resultados de la relación entre ambas partes, así como con la vinculación afectiva entre las metas y valores de la organización y el empleado (Buchanan, 1974), afectando al incremento del desempeño laboral, al ausentismo ${ }^{1}$ y a la rotación laboral (Betanzos y Paz, 2007).

\section{Metodología}

La investigación propuesta en este documento se apoya en el análisis bibliométrico de las publicaciones científicas, donde como unidad básica se utiliza el artículo científico. La decisión de utilizar esta metodología se justifica por la capacidad de visualizar de forma pragmática y sencilla la evolución sobre los temas de estudios en cuestión. En cuanto a cómo se ha llevado a cabo el análisis, se ha realizado un detalle pormenorizado en dos conjuntos diferenciados, indicadores de actividad e indicadores de relación. La observación y estudio de los artículos seleccionados revelan cuál ha sido la evolución de las investigaciones relacionadas con el contrato psicológico y el compromiso organizacional. Asimismo, la información extraída de los artículos a través del año de publicación, autores, citas, procedencia de los artículos, índices de impacto, y otras características, permite realizar un completo esquema de lo que ha significado la producción científica en este ámbito.

$\mathrm{El}$ análisis de publicaciones realizado con el fin de encontrar artículos relacionando el contrato psicológico y el compromiso organizacional se ha efectuado utilizando como parámetros de búsqueda las expresiones psychological contract y

$1 \mathrm{El}$ autor hace referencia al absentismo. 
organizational commitment. El sondeo se ha efectuado con los mismos ítems en dos bases de datos: Web Of Science y Scopus, ambas disponibles en la página web del Ministerio de Ciencia, Innovación y Universidades.

En la base Web of Science se ha restringido la búsqueda a los índices Science Citation Index Expanded (SCI-EXPANDED) y Social Sciences Citation Index (SSCI). En ambos casos no se limita el año de publicación. La búsqueda se realiza buscando coincidencias de psychological contract y organizational commitment, tanto en el título como en el resumen o abstract y en las palabras clave o keywords de los artículos disponibles en la base de datos. Para afinar un poco más la búsqueda respecto a los ítems seleccionados, se restringe a las categorías Management, Phychology applied, Business y Sociology. Esta configuración de búsqueda selecciona un total preliminar de 220 artículos en esta fuente (febrero 2019).

En la base Scopus se realiza la misma búsqueda que en Web of Science, psychological contract y organizational commitment para los campos título, resumen y palabras clave. Además, se limita el resultado a las familias Business, Management and Accounting, Social Sciencies y Psychology. En este caso, la configuración de la búsqueda localiza un total de 152 artículos en Scopus (febrero 2019).

Una vez realizadas las búsquedas comentadas, se obtienen 372 artículos, resultado de sumar los listados de las dos bases. A partir de aquí, y con el fin de concentrar el análisis a los artículos más relevantes, se han seleccionado únicamente aquellos que muestren un número de citas igual o superior a 5 . De este modo se obtienen 170 artículos con 5 citas o más en WOS (Web of Science) y 100 en Scopus. A partir de este conjunto reducido a 270 unidades, se realiza un cruce entre las dos listas conseguidas para evitar duplicidades. Tras la comprobación, la selección final objeto de este estudio se centra en 207 artículos, procedentes de 86 publicaciones distintas, 28 países y más de 300 autores.

La bibliometría cuenta con diversos métodos y técnicas para representar y conocer la evolución y actualidad de una disciplina científica determinada. Estos análisis bibliométricos se pueden dividir en dos categorías principales según las herramientas empleadas: aquellas que utilizan indicadores de actividad y las que emplean indicadores de relación (Callon, Courtial y Penan, 1995). Las primeras arrojan datos de carácter cuantitativo, principalmente, acerca del volumen, impacto y etapa de las actividades de investigación, mientras que las segundas se centran sobre las relaciones temáticas que hay entre los investigadores y sus investigaciones, así como la evolución de los contenidos.

Los indicadores de actividad, como herramienta bibliométrica, que se describen en el apartado siguiente, permitirán dibujar una guía para que el lector se pueda hacer una idea clara de los aspectos cuantitativos relacionados con las publicaciones sobre contrato psicológico y su relación con el compromiso or- 
ganizacional. El número de artículos de este campo, relacionados con el año y país de publicación, así como la productividad de los diferentes investigadores e instituciones situarán en un panorama temporal el estado de esta temática y su evolución en el tiempo.

Los indicadores relacionales que se desarrollarán a continuación aportarán un análisis sobre el contenido de los artículos seleccionados. De este modo, y mediante un análisis de copalabras y de coocurrencias, se representará el estado actual de la disciplina, así como un recorrido según las tendencias por temas y países de los últimos años. Estas técnicas permitirán a través de los resultados obtenidos mostrar las relaciones entre los temas estudiados y posibilitarán la identificación de líneas emergentes de investigación (Courtial, 1994, citado por Benavides, Guzmán y Quintana, 2011).

En resumen, el proceso de búsqueda en las dos bases de datos comentadas de los términos psychological contract y organizational commitment, contenidos en el título, resumen o palabras clave (incluso en el texto completo), ha permitido llegar a un conjunto inicial de 372 artículos. Posteriormente se realiza un filtrado en varias fases; selección de aquellos artículos con un mínimo de cinco citas; eliminación de artículos duplicados en las dos bases consultadas, y comprobación de que el contenido del artículo se ajusta a la temática buscada. Esto ha propiciado un número final de 207 artículos consultados, que, tras la aplicación de técnicas bibliométricas mediante indicadores de actividad y relacionales, permite una serie de resultados que se detallan en los apartados siguientes.

\section{Indicadores de actividad en la literatura sobre contrato psicológico y compromiso}

La evolución del número de publicaciones por año ha experimentado un notable aumento. La muestra que se analiza consta de 207 artículos. El primero de ellos aparece en 1991, "The institutionalization of organizational ethics", publicado por Sims en Journal of Business Ethics. Esta revista pionera permanece como una de las más productivas en esta temática, si bien su contribución no guarda un ritmo constante.

Hasta el año 2000 incluido, el número de investigaciones publicadas dentro de la muestra escogida fue solo de 18 unidades (casi un 9\%). Entre el año 2001 y 2010 este número asciende a 103 artículos, y desde 2011 hasta nuestros días se han publicado en dichas fuentes y con un mínimo de cinco citas un total de 86 trabajos. Teniendo en cuenta que a esta década aún le faltan dos años de producción y que los artículos citados ganan protagonismo en los años sucesivos 
a su publicación, se puede destacar que se ha producido un aumento considerable del ritmo de publicación relativa a esta temática.

Gráfico 1. Número de artículos por año de publicaciónFuente‡ elaboración propia a partir de datos de WOS y Scopus.

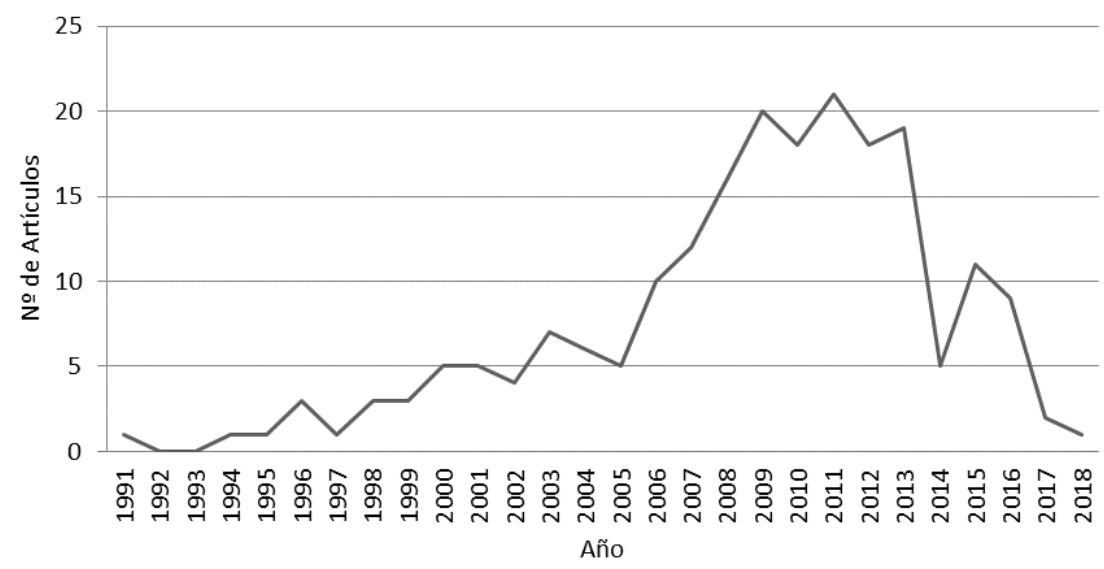

Fuente: elaboración propia a partir de datos de WOS y Scopus

El Gráfico 1 muestra cómo a partir de 2005 el crecimiento de publicaciones es muy elevado, manteniéndose un ritmo alrededor de 18 artículos por año hasta 2013, donde se aprecia una caída pronunciada. Hasta el año 2000 solo investigadores de los Estados Unidos de América e Inglaterra habían publicado de forma significativa, Canadá, Holanda, Francia y Suiza aparecen en este período solo con un artículo. En esta fase, antes del cambio de siglo, sobresale la investigación realizada por Guzzo, Noonan y Elron titulada "Expatriate managers and the psychological contract" (1994), que se convierte en una referencia para trabajos posteriores.

En la recopilación y recuento de artículos por autor se encuentran grupos de investigadores que han publicado de forma conjunta un número considerable de artículos. En este estudio bibliométrico nos surtimos de dos bases de datos distintas. Hay autores y publicaciones que aparecen en ambas bases, siendo este el caso más común. Cada base de datos (WOS o Scopus) puntúa y publica el índice de Hirsch, o índice h de cada autor. La relación entre el número de publicaciones y las veces que ha sido citado es diferente según la base que consultemos. No obstante, se mantiene cierta proporcionalidad entre los indicadores de ambas bases; en la tabla 1 se aprecian los autores con más número de publicaciones y el índice h en cada base de datos. 
Tabla 1. Autores que han publicado más de dos artículos sobre Contrato Psicológico y Compromiso Organizacional

\begin{tabular}{|l|c|c|c|c|}
\hline Autor & $\begin{array}{c}\text { Número de } \\
\text { artículos }\end{array}$ & $\begin{array}{c}\text { Último artículo } \\
\text { publicado }\end{array}$ & $\begin{array}{c}\text { Índice h en } \\
\text { WOS }\end{array}$ & $\begin{array}{c}\text { Índice h en } \\
\text { Scopus }\end{array}$ \\
\hline De Witte, H. & 10 & 2016 & 39 & 19 \\
\hline De Cuyper, N. & 8 & 2016 & 28 & 31 \\
\hline Ng, T. W. H. & 7 & 2015 & 33 & 35 \\
\hline Feldman, D. C. & 7 & 2012 & 52 & 54 \\
\hline Bal, P. M. & 6 & 2016 & 17 & 20 \\
\hline Schalk, R. & 5 & 2012 & 20 & - \\
\hline Conway, N. & 4 & 2011 & 18 & 19 \\
\hline De Lange, A. H. & 4 & 2015 & 22 & 21 \\
\hline Guest, D. & 4 & 2011 & 30 & 38 \\
\hline Buttner, E. H. & 3 & 2010 & 10 & 13 \\
\hline Solinger, O. N. & 3 & 2016 & 5 & 6 \\
\hline Bellou, V. & 2 & 2009 & 8 & 10 \\
\hline Bernhard-Oettel, C. & 2 & 2013 & 8 & 9 \\
\hline Chang, E. M. & 2 & 2009 & 12 & - \\
\hline Coyle-Shapiro, J. A. M. & 2 & 2006 & 16 & 22 \\
\hline Crawshaw, J. R. & 2 & 2015 & 6 & \\
\hline Freese, C. & 2 & 2011 & 5 & 7 \\
\hline Massingham, P. R. & 2 & 2018 & 8 & - \\
\hline Meyer, J. P. & 2 & 2010 & & 42 \\
\hline Morrell, K. & 2 & 2004 & 13 & - \\
\hline Parzefall, M. R. & 2 & 2012 & 6 & 20 \\
\hline Richard, O. C. & 2 & 2009 & 16 & - \\
\hline Rodwell, J. J. & 2 & 2011 & 17 & 21 \\
\hline Sora, B. & 2 & 2010 & 8 & \\
\hline Sturges, J. & 2005 & 17 & \\
\hline & & & & \\
\hline
\end{tabular}

Fuente: elaboración propia a partir de datos de WOS y Scopus.

Es preciso comentar que el autor con más publicaciones (10), De Witte, solo aparece como primer autor en un artículo de la decena donde participa. En cambio, De Cuyper (8) sí es el autor que figura primero en los artículos con su firma. Los autores más citados por artículo no están representados en la lista anterior, pues su producción en este campo no ha sido tan numerosa. El artículo más citado de todos los analizados corresponde a Zhao, Wayne, Glibkowski y Bravo, que en 2007 publican "The impact of psychological contract breach on work-related outcomes: A meta-analysis", y que cuenta con 517 citas de otros investigadores.

Respecto a las revistas con mayor presencia en las investigaciones sobre contrato psicológico y compromiso organizacional, se puede indicar que el conjunto 
de los 207 artículos estudiados en este trabajo desde 1991 a 2018 se reparten en 86 publicaciones distintas, destacando las más representativas con 20 y 15 investigaciones editadas. Al igual que ocurre con la producción investigadora en este campo, las revistas más activas aparecen en esta temática a partir de 2005, publicando de manera casi continua un significativo número de artículos cada año.

El percentil de factor de impacto de las revistas principales de este bibliométrico aparece situado habitualmente en el primero y en el segundo cuartil. En la tabla 2 se detallan las revistas con más incidencia en los estudios sobre contrato psicológico y compromiso organizacional. Tanto en la base de datos WOS, a través de sus familias Science Citation Index Expanded (SCI-EXPANDED) y Social Sciences Citation Index (SSCI), como en la base de datos Scopus, se muestran los cuartiles respecto al resto de publicaciones en cada categoría temática y cada base. Hay casos donde alguna revista presenta una clasificación diferente según distintas áreas de conocimiento, en este caso se ha elegido la correspondiente a la temática que nos ocupa. El resultado de la exploración de las revistas se marca con una"S" (Scopus) o una "W" (WOS) en el cuartil correspondiente.

Tabla 2. Revistas y clasificación por cuartiles sobre contrato psicológico y compromiso organizacional

\begin{tabular}{|c|l|c|c|c|c|c|c|}
\hline N.* & Revistas & Q1 & Q2 & Q3 & Q4 & $\begin{array}{c}\text { Artículos } \\
\text { totales }\end{array}$ & $\%$ \\
\hline 1 & $\begin{array}{l}\text { International Journal of Human Resource } \\
\text { Management }\end{array}$ & $\mathrm{S}$ & $\mathrm{W}$ & & & 20 & 9,7 \\
\hline 2 & Journal of Vocational Behavior & $\mathrm{W}$ & & & & 15 & 7,2 \\
\hline 3 & Journal of Organizational Behavior & $\mathrm{WS}$ & & & & 9 & 4,3 \\
\hline 4 & Personnel Review & & & $\mathrm{WS}$ & & 8 & 3,9 \\
\hline 5 & Human Relations & $\mathrm{WS}$ & & & & 7 & 3,4 \\
\hline 6 & Journal of Business Ethics & $\mathrm{WS}$ & & & & 7 & 3,4 \\
\hline 7 & Journal of Managerial Psychology & $\mathrm{S}$ & & $\mathrm{W}$ & & 6 & 2,9 \\
\hline 8 & Career Development International & $\mathrm{S}$ & $\mathrm{W}$ & & & 5 & 2,4 \\
\hline 9 & Economic and Industrial Democracy & & & $\mathrm{W}$ & & 5 & 2,4 \\
\hline 10 & Journal of Applied Psychology & $\mathrm{W}$ & & & & 5 & 2,4 \\
\hline 11 & Employee Relations & & $\mathrm{WS}$ & & & 4 & 1,9 \\
\hline 12 & $\begin{array}{l}\text { European Journal of Work and Organiza- } \\
\text { tional Psychology }\end{array}$ & $\mathrm{W}$ & & & & 4 & 1,9 \\
\hline 13 & Group E Organization Management & $\mathrm{W}$ & & & & 4 & 1,9 \\
\hline 14 & International Journal of Manpower & $\mathrm{S}$ & & & $\mathrm{W}$ & 4 & 1,9 \\
\hline 15 & Journal of Business and Psychology & $\mathrm{W}$ & & & & 4 & 1,9 \\
\hline 16 & Journal of Business Research & & $\mathrm{W}$ & & & 4 & 1,9 \\
\hline 17 & $\begin{array}{l}\text { Journal of Occupational and Organization- } \\
\text { al Psychology }\end{array}$ & $\mathrm{W}$ & & & & 4 & 1,9 \\
\hline
\end{tabular}

Fuente: elaboración propia a partir de datos de WOS y Scopus. 
La International Journal of Human Resource Management, cuyo volumen 1 aparece en el año 1990, se muestra como la más activa en este campo, con 20 artículos desde 2003, y con una continua producción hasta estos días. La segunda publicación, con 15 artículos, Journal of Vocational Behavior, aparece en 1971, y, al igual que la anterior, a partir de principios del siglo xxI recoge una serie de investigaciones sobre la temática que nos ocupa, si bien, como ocurre en el cómputo general, demuestra más actividad a partir de 2008. En la tabla 3 se muestran de nuevo las revistas que cuentan al menos con cuatro publicaciones dentro de la selección realizada en este artículo y su actividad por años.

Como se puede apreciar, ninguna revista representa más de un 10\% de los artículos publicados, lo que indica que ninguna revista se ha especializado específicamente en esta materia; también da una idea de cómo la temática que nos ocupa queda representada desde enfoques multidisciplinares, y que, aun habiendo acotado la búsqueda en determinadas áreas de conocimiento, el estudio del contrato psicológico y el compromiso organizacional admite puntos de vista desde variadas disciplinas.

Tabla 3. Actividad anual de revistas en artículos sobre contrato psicológico y compromiso organizacional

\begin{tabular}{|c|c|c|c|c|c|c|c|c|c|c|c|c|c|c|c|c|c|c|c|c|c|c|c|c|c|c|c|c|}
\hline & ఏ & ఏँ & 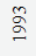 & 总 & ळ & ఏ & ఏ & $\stackrel{\infty}{\circ}$ & ఏे & ڤ్ & চ্ণ & ڤ్ & ڤ్ & ڤ్̀ & ڤ్ & Бั่ & స్ స్ & ڤัे & ڤેे & $\stackrel{\circ}{\circ}$ & $\overline{\widetilde{\sigma}}$ & స̃ & $\stackrel{m}{\tilde{\Xi}}$ & $\stackrel{d}{\stackrel{d}{\sim}}$ & $\stackrel{n}{\bar{c}}$ & 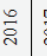 & & $\stackrel{\infty}{\tilde{\sigma}}$ \\
\hline 1 & & & & & & & & & & & & & & & & & & & & & & & . & & & & & \\
\hline 2 & & & & & & & & & & & & & & & & & & & & & & & & & & & & \\
\hline 3 & & & & & & & & & & & & & & & & & & & & & & & & & & & & \\
\hline 4 & & & & & & & & & & & & & & & & & & & & & & & & & & & & \\
\hline 5 & & & & & & & & & & & & & & & & & & & & & & & & & & & & \\
\hline 6 & & & & & & & & & & & & & & & & & & & & & & & & & & & & \\
\hline 7 & & & & & & & & & & & & & & & & & & & & & & & & & & & & \\
\hline 8 & & & & & & & & & & & & & & & & & & & & & & & & & & & & \\
\hline 9 & & & & & & & & & & & & & & & & & & & & & & & & & & & & \\
\hline 10 & & & & & & & & & & & & & & & & & & & & & & & & & & & & \\
\hline 11 & & & & & & & & & & & & & & & & & & & & & & & & & & & & \\
\hline 12 & & & & & & & & & & & & & & & & & & & & & & & & & & & & \\
\hline 13 & & & & & & & & & & & & & & & & & & & & & & & & & & & & \\
\hline 14 & & & & & & & & & & & & & & & & & & & & & & & & & & & & \\
\hline 15 & & & & & & & & & & & & & & & & & & & & & & & & & & & & \\
\hline 16 & & & & & & & & & & & & & & & & & & & & & & & & & & & & \\
\hline 17 & & & & & & & & & & & & & & & & & & & & & & & & & & & & \\
\hline
\end{tabular}

Fuente: elaboración propia a partir de datos de WOS y Scopus.

La producción bibliográfica por países, tomando como referencia el lugar de trabajo del investigador, arroja una mayoría destacada de Estados Unidos, con 61 artículos. Le sigue con menos de la mitad Inglaterra (27), y después, Australia (16), Holanda (13), Canadá y China (12), Bélgica (11), España (7), Alemania y Taiwán (6), Francia y Grecia (5), Suecia (4), India (3), Israel, Malasia, Sudáfrica, 
Emiratos Árabes Unidos y Gales (2), y, por último, Indonesia, Italia, Japón, Pakistán, Portugal, Escocia, Corea del Sur, Suiza y Turquía (1).

La producción en este campo en el caso de España, con siete artículos, se distribuye entre los años 2008 y 2013. Cantisano, Domínguez y Depolo (2008) son los primeros en publicar respecto a este tema. Destacan a partir de 2009 los trabajos de Sora, Caballer y Peiro (2009 y 2010), que realizan dos trabajos, uno de ellos junto con Witte (2009), que es el autor más prolífico de este estudio. Simó y Enache también aparecen con dos publicaciones en 2010 y 2013. Silla, Gracia, Manas y Peiro (2010) hacen una aportación interesante sobre las relaciones entre inseguridad laboral y equidad en el trabajo. La última publicación registrada en el momento de realizar este análisis es la de Susaeta, Pin, Idrovo, Espejo y Belizón (2013), que realizan un análisis sobre diferentes países iberoamericanos al objeto de conocer qué efectos tienen la generación y la cultura en las actitudes de los trabajadores.

\section{Evolución de la investigación sobre contrato psicológico y compromiso organizacional}

Este artículo pretende describir las tendencias más importantes de la literatura sobre trabajos en los que, en un contexto laboral, se estudien el contrato psicológico y el compromiso organizacional como factores directamente relacionados entre sí o formando parte de análisis transversales. No existe una categorización generalmente aceptada que englobe las temáticas tratadas sobre la investigación de la conexión de estos dos constructos teóricos. De nuestra selección de artículos (Figura 1), se ha desprendido una categorización en función del enfoque de los trabajos analizados. A la hora de categorizarlos se ha tenido en cuenta la temática central de la investigación en relación con sus objetivos y sus resultados, de esta manera, se encuentra que en el eje "contrato psicológico y compromiso organizacional" existen diversas formas de abordar esta temática; por su centralidad, la primera es aquella que entronca la relación de ambos conceptos como principal objetivo de la investigación, sin embargo, encontramos trabajos en los que se analizan de forma separada. Uno de los enfoques más importantes en cuanto a las citas que acumulan los trabajos y la diversidad de los temas tratados es el que ha estudiado los aspectos organizacionales de la empresa, con temas que abordan, entre otros, la inseguridad laboral, el papel del supervisor en el compromiso organizacional, la justicia o la ética organizacional. 
Figura 1. Enfoques de investigación en el eje contrato psicológico y compromiso organizacional

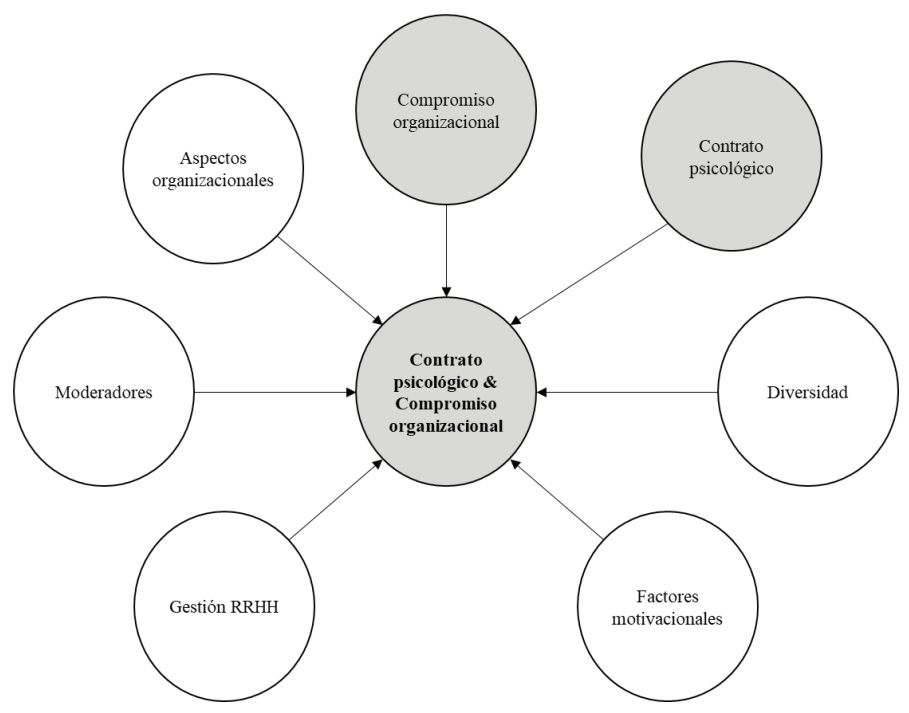

Fuente: elaboración propia

En la revisión de la literatura objeto de este estudio, se aprecia otro enfoque que engloba trabajos que tienen que ver con la gestión de $R R$. HH. y que relacionan el contrato psicológico y el compromiso organizacional con la carrera profesional, la externalización de los RR. HH., la formación, la innovación, las prácticas o los diversos enfoques estratégicos de RR. HH., entre otros. Por otra parte, existen trabajos que han abordado sus investigaciones sobre esta temática desde la aplicación de diferentes moderadores, tales como la tipología de empleo, moderadores demográficos, análisis desde la perspectiva de trabajadores expatriados e impatriados, la repercusión de los factores personales o los análisis generacionales. Por último, se han encontrado trabajos en los que el contrato psicológico y el compromiso organizacional se relaciona con la gestión de la diversidad y los factores motivacionales, con temas como la etnia y la raza, la interculturalidad y la multiculturalidad, o los factores motivacionales intrínsecos, sentimientos hacia la organización o factores de contención.

Los primeros trabajos que se publican relacionados con el contrato psicológico y el compromiso organizacional se encuentran entre 1991 y 1995. Por una parte, se centran en la ética organizacional y su institucionalización en las organizaciones (Sims, 1991; Carlson y Perrewe, 1995), y, por otra, en la investigación sobre las prácticas de RR. HH, puestas en marcha por las empresas en relación con los gerentes expatriados y su repercusión con el compromiso organizacional 
y la intención de volver a su país de origen, investigando el contrato psicológico como mediador (Guzzo, Noonan y Elron, 1994). En cuanto a los niveles de producción científica relacionados con la temática principal (Tabla y gráfico 2), en los primeros quince años (1991-2005) se aborda apenas el 22\% del total de artículos del período muestral. Se inauguran los trabajos relacionados con los dos temas troncales, $\mathrm{CP}$ y $\mathrm{CO}$, con desencuentro en sus conclusiones. Millward y Hopkins (1998) publican el primer trabajo haciendo referencia a la aportación de Rousseau (1995) sobre la tipología de los contratos relacional y transaccional. Millward y Hopkins (ibid.) tratan de evidenciar la relación de estos con el compromiso organizacional, de tal manera que señalan que "cuanto más relacional sea la orientación psicológica de los empleados, mayor será su nivel de compromiso organizacional, por el contrario, cuanto más transaccional sea la orientación psicológica de los empleados, menor será su nivel de compromiso organizacional“. Sin embargo, McDonald y Makin (2000) analizan la misma cuestión y sugieren contradicciones con la tesis anterior, planteando que apenas hay diferencia en el comportamiento de los trabajadores que tienen un tipo de contrato $\mathrm{u}$ otro, señalando, incluso, que el nivel de satisfacción y compromiso es mayor en los trabajadores de contrato transaccional.

Los años más fructíferos en cuanto a producción científica relacionada con la temática objeto de este trabajo se dan entre 2006 y 2015, con el $72 \%$ de toda la muestra. En estos años, existe un alto interés por trabajos que tienen que ver con el enfoque aspectos organizacionales, concretamente relacionados con inseguridad laboral, el papel del supervisor en el compromiso organizacional y la justicia organizacional ${ }^{2}$. También irrumpen una serie de trabajos coincidiendo con los años de crisis, en los que se investiga sobre los cambios en la organización, es decir, cómo repercuten estos cambios en el contrato psicológico y en el compromiso organizacional. Los trabajos que estudian de forma separada el contrato psicológico tienen su mayor presencia entre los años 2006 y 2010, para luego ir bajando progresivamente; los temas más investigados son los relacionados con el desenlace del contrato psicológico y los que estudian los contratos psicológicos relacionales y transaccionales. Por otra parte, aquellos que analizan el contrato psicológico $y$ compromiso organizacional conjuntamente arrancan en 2001 y mantienen su interés de forma creciente hasta 2015. En cuanto a los trabajos relacionados con el compromiso organizacional como tema principal, aunque en menor medida que los anteriores, se han mantenido constantes en el período 2001-2015 y se han centrado en temas como la rotación o el análisis de factores que influyen en el compromiso organizacional.

2 Véase el anexo I. 
Teniendo en cuenta que para la selección de artículos se han elegido aquellos que hayan sido citado al menos cinco veces, encontramos que en el último tramo, de 2016 a 2018, hay muchos menos trabajos que en períodos anteriores. Los enfoques sobre los que se orientan estas publicaciones parecen seguir la misma senda, salvo en investigaciones sobre la diversidad y factores motivacionales, sobre los que no se han encontrado publicaciones en este período.

\section{Tabla 4. Evolución trabajos de investigación sobre CP y CO}

\begin{tabular}{|c|c|c|c|c|c|c|c|}
\hline Enfoque de la investigación & $\begin{array}{l}\frac{2}{2} \\
\frac{1}{2} \\
\frac{1}{2}\end{array}$ & 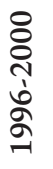 & 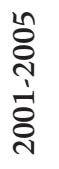 & 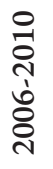 & 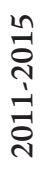 & 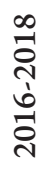 & ڤ్ \\
\hline Aspectos organizacionales & 2 & 4 & 6 & 22 & 25 & 4 & 63 \\
\hline Moderadores & 1 & 3 & 7 & 12 & 10 & 2 & 35 \\
\hline Gestión de RR.HH. & 0 & 5 & 4 & 12 & 10 & 1 & 32 \\
\hline Contrato psicológico (CP) & 0 & 2 & 0 & 12 & 7 & 2 & 23 \\
\hline $\mathrm{CP}$ y CO conjuntamente & 0 & 0 & 2 & 7 & 12 & 1 & 22 \\
\hline $\begin{array}{l}\text { Compromiso organizacional } \\
\text { (CO) }\end{array}$ & 0 & 1 & 6 & 6 & 5 & 2 & 20 \\
\hline Diversidad & 0 & 0 & 1 & 4 & 2 & 0 & 7 \\
\hline \multirow[t]{2}{*}{ Factores motivacionales } & 0 & 0 & 1 & 1 & 3 & 0 & 5 \\
\hline & 3 & 15 & 27 & 76 & 74 & 12 & 207 \\
\hline
\end{tabular}

Fuente: elaboración propia.

\section{Gráfico 2.}

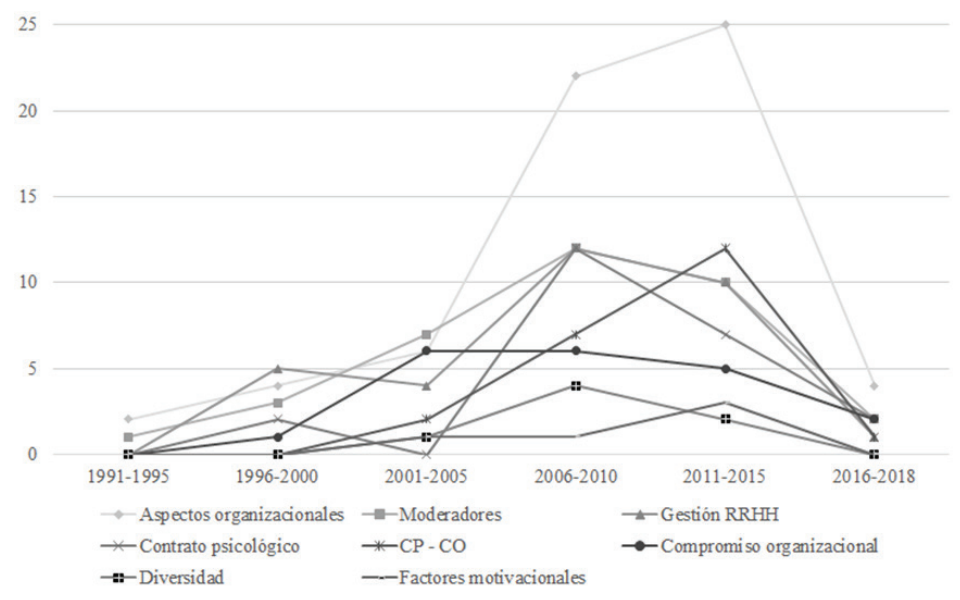

Fuente: elaboración propia 


\section{Principales investigaciones sobre contrato psicológico y compromiso organizacional}

\section{a) Compromiso organizacional}

Desde la perspectiva de intercambio social, y más en concreto con la capacidad de contribución individual y reciprocidad de la organización, la literatura sugiere, a través de determinados trabajos, que la participación en las ganancias altera de forma positiva el compromiso organizacional y la confianza en la gestión (CoyleShapiro, Morrow, Richardson y Dunn, 2002). Por otra parte, otros temas relacionados con el compromiso organizacional, como es la rotación o la aceptación de la cultura organizacional, en la que Porter y Lawer (1965) hacen especial énfasis al referirse al anhelo de permanecer en esta y aceptar sus principales objetivos y valores, la investigación se ha centrado, fundamentalmente, en el sentido de la necesidad de desarrollar políticas e intervenciones dirigidas a la creación de entornos de trabajo en los que se generen altos niveles de confianza y satisfacción por parte del trabajador, permitiendo potenciar y mejorar el compromiso organizacional (Gregory, Way, LeFort, Barrett y Parfrey, 2007). Por otra parte, en cuanto a la rotación voluntaria, los resultados de un trabajo de $\mathrm{Ng}$ y Feldman (2009) sugieren que, si bien la literatura apunta a que la edad y la rotación voluntaria están débilmente relacionadas, la movilidad entre los empleados en las dos décadas anteriores puede haber sufrido cambios en el sentido de que la relación era más fuerte, sobre todo cuando en la muestra se daba un mayor número de casos de minorías raciales o menor nivel educativo. Por otra parte, con respecto a la aceptación de valores o cultura organizacional, la literatura apoya la tesis de que la cultura de la organización juega un papel importante en el nivel de satisfacción en el trabajo y el compromiso organizacional; así, en una organización burocratizada se dan niveles más bajos de compromiso que en una cultura centrada en el apoyo (Silverthorne, 2004).

\section{b) Contrato psicológico y compromiso organizacional}

Con respecto a la investigación del contrato psicológico, en la revisión de la literatura, el mayor número de trabajos se centraban en analizar el desenlace o efectos de la ruptura o violación y el cumplimiento del contrato psicológico, y sobre las tipologías de contrato relacional y contrato transaccional, alumbrados por Rousseau (1995). Si bien existen trabajos que hacen distinción entre ruptura y violación del contrato psicológico (Topa y Palací, 2004), no hay una aclaración aceptada que permita diferenciar dichos términos a lo largo de los trabajos revisados; sin embargo, la literatura sí trata de diferenciar entre los efectos de la rup- 
tura o violación y los efectos del cumplimiento. En este sentido, Conway, Guest y Trenberth (2011) sugieren que los efectos de la ruptura tienen importantes consecuencias negativas sobre el bienestar y la mejora de actitudes, sin embargo, apenas son perceptibles los efectos positivos del cumplimiento; por el contrario, con respecto a esta última afirmación, Parzefall y Hakanen (2010) asocian la percepción del cumplimiento del contrato psicológico con una mayor motivación y una mejora de la salud y del compromiso organizacional.

En cuanto a los factores que pueden provocar la ruptura del contrato psicológico, existen en el contexto organizacional o laboral situaciones a) de tipo organizativo, tales como la falta de autonomía y participación, la falta de claridad en los objetivos organizacionales, la escasez de apoyo emocional, la inseguridad laboral, o b) de tipo personal o intrínseco, como el incumplimiento de planes de formación, la falta de oportunidades para la carrera profesional o movilidad ascendente. A su vez, la ruptura del contrato psicológico desencadena efectos negativos que provocan bajos niveles de satisfacción, quebranto del compromiso organizacional, baja participación en el trabajo, resultados negativos en el desempeño o la intención de abandonar el empleo (Granrose y Baccili, 2006). Otras investigaciones señalan que los efectos negativos de la ruptura del contrato psicológico pueden verse atenuados con el apoyo social aportado por los mentores internos que tutorizan a los empleados, también que la inacción de dichos mentores provoca el efecto contrario, por lo tanto, sugieren alentar a las organizaciones para proporcionar capacitación a los mentores potenciales en la gestión de la relación de tutoría para garantizar los efectos deseados (Haggard, 2012). Finalmente, Tomprou, Rousseau y Hansen (2015) diseñan un modelo posviolación del contrato psicológico teniendo en cuenta que los resultados de este dependen de las experiencias posteriores a la violación de la víctima y las estrategias de afrontamiento. El modelo contempla cuatro formas en las que este puede resolverse: a) reactivación del contrato psicológico, que se produce cuando este se devuelve a su estado previo a la violación, lo que permite al empleado que confíe una vez más en él para guiar las percepciones y comportamientos; b) el contrato psicológico prospera, que ocurre cuando la víctima acepta un contrato revisado, que es más beneficioso para él o ella que el contrato previo a la violación; c) deterioro psicológico del contrato, que ocurre cuando el empleado llega a aceptar un contrato revisado que es menos beneficioso que el contrato previo a la violación, y d) la disolución del contrato psicológico, que sucede cuando la víctima no puede aceptar las circunstancias alteradas como una base para un nuevo contrato psicológico, eligiendo permanecer en su lugar en un estado crónico y disfuncional de violación sentida. En relación con este modelo, Solinger, Hofmans, Bal y Jansen (2016) 
evidencian que el impacto emocional de la violación y el apoyo organizacional percibido después están relacionados con el éxito del proceso de resolución de incumplimiento. Además, revelan un componente no lineal en las trayectorias postincumplimiento de compromiso que sugiere que los procesos que determinan el éxito de la resolución de incumplimiento son más complejos de lo que actualmente se asume.

Con respecto la investigación de los contratos de tipo relacional y transaccional, como se ha señalado anteriormente, la literatura plantea que las organizaciones utilizan personal permanente, al que se le supone un contrato relacional, y esto implica niveles más altos de compromiso con la organización y personal no permanente como un recurso flexible, de carácter temporal, y con un menor compromiso organizacional, al que se le atribuye un contrato transaccional (Rousseau, 1995; Arnold, 1996; Millward y Hopkins, 1998); sin embargo, en la revisión realizada de la bibliografía, aunque se asume que en este tipo de contratos existen bajos niveles de compromiso con la organización, los niveles de compromiso con los compañeros, supervisores y, en algunos casos, los clientes de la propia organización se mantienen altos (Ainsworth y Purss, 2009). Otras líneas de investigación han analizado el estrés laboral en los trabajadores temporales contrato transaccional-, concluyendo que estos sufren un mayor nivel de estrés y de tensión en el trabajo, lo cual desencadena una percepción de violación del contrato psicológico, afectando de forma negativa al compromiso organizacional (Yeh, Ko, Chang y Chen, 2007). Por otra parte, desde el análisis de la cultura organizacional, existen trabajos que argumentan que los contratos transaccionales están más relacionados con las culturas jerárquicas y los contratos relacionales son más dados en culturas organizacionales que tienen una mayor cohesión tipo camarilla o clan (Richard, McMillan-Capehart, Bhuian y Taylor, 2009). Otros trabajos de investigación han profundizado en el análisis del contrato psicológico transaccional que se da en organizaciones con personal externo o contratado en empresas de trabajo temporal. Estas sugieren que, teniendo en cuenta que el incumplimiento del contrato psicológico de los trabajadores temporales puede provenir de parte de la empresa de trabajo temporal o la empresa cliente, la relación negativa con el compromiso es hacia la parte que incumple (Lapalme, Simard y Tremblay, 2011); por otra parte, en el sentido del cumplimiento de contrato, Coyle-Shapiro y Morrow (2006) señalan que la percepción de apoyo organizacional de la empresa cliente y el atractivo de la empresa en sí mismo se relacionan positivamente con el compromiso afectivo de los empleados hacia la organización del cliente, y que el apoyo de la empresa externa o de trabajo temporal también explica la variación del compromiso afectivo hacia el cliente, lo que sugiere que 
los empleados que se sienten apoyados por su propia organización contratante se sienten más comprometidos con la organización del cliente.

\section{c) Cuestiones poco investigadas}

Una vez revisada la literatura de la muestra sobre los conceptos objeto de este trabajo, nos encontramos con que existen cuestiones de especial relevancia, unas por el actual interés social (diversidad o igualdad de género) y otras por la manera en que pueden influir sobre el compromiso organizacional y el contrato psicológico (cultura organizacional), que están escasamente investigadas.

Con respecto a la diversidad, solo se han encontrado tres publicaciones relacionadas con etnia y raza, todas de Buttner, Lowe y Billings-Harris, entre 2010 y 2012. Investigan sobre la conciencia racial, el clima de la diversidad y qué efecto tienen sobre la violación del contrato psicológico. En cuanto a multiculturalidad e interculturalidad, apenas se han encontrado cuatro trabajos. En el primero, Gelade, Dobson y Auer (2008) analizan 29 naciones, y a partir de las dimensiones de Hofstede tratan de explicar los efectos de la cultura nacional sobre el compromiso organizacional. Magoshi y Chang (2009) centran una investigación en Japón y Corea. Plantean la importancia de la gestión de la diversidad como recurso competitivo y sus efectos positivos sobre el compromiso organizacional. Por otra parte, Kickul, Lester y Belgio (2004) estudian, a partir de una muestra de empleados americanos y chinos de Hong Kong, cómo afecta la diferencia cultural a las percepciones sobre el contrato psicológico, satisfacción en el trabajo, intención de abandonar el trabajo, compromiso organizacional y el comportamiento de la ciudadanía organizativa. Por último, Susaeta, Piñeiro, Idrovo Carlier, Espejo y Belizón (2013) analizan diversos países iberoamericanos con el propósito de encontrar evidencias de los efectos que tienen en las actitudes de los trabajadores la pertenencia a una generación y a una cultura determinada.

En cuanto a la literatura relacionada con género, cinco trabajos encierran toda la investigación. Todos utilizan género como moderador. Cheng y Chan (2008) y Keim, Landis, Pierce y Earnest (2014), a través de metaanálisis, investigan la inseguridad laboral; Bellou (2019) analiza la percepción de contrato psicológico deseable, entre otros factores, por razones de género; Seong, Hong y Park (2012) hacen un análisis en Corea en el que pretenden examinar la relación entre trabajador y organización teniendo en cuenta género y tipo de contrato, $y$, por último, Willem, De Vos y Buelens (2010) hacen alusión al género en un estudio en el que tratan de comparar la percepción del contrato psicológico en trabajadores del sector público frente a los del sector privado, sugiriendo que existen diferencias 
significativas de género en cuanto a la percepción de la importancia del cumplimento del contrato psicológico.

Por último, en cuanto a la producción de trabajos de investigación sobre cultura organizacional, en la muestra solo se han encontrado dos publicaciones. En la primera, Silverthorne (2004) trata de explicar la importancia que tiene la cultura organizacional en el ajuste entre personas y organización, y cómo repercute en los niveles de satisfacción y compromiso organizacional, y en la segunda, Welch y Welch (2006) analizan la eficacia de la cultura corporativa como mecanismo de control en el contexto multinacional, sugiriendo que a largo plazo a las multinacionales no les interesa tener una cultura corporativa fuerte.

\section{Conclusiones}

La literatura relacionada con contrato psicológico y compromiso organizacional ha ido produciendo trabajos de forma independiente aportando un valioso conocimiento de cara a afrontar de mejor manera la eficiencia dentro del contexto organizacional. El objetivo de este trabajo era conocer más sobre estos conceptos de manera relacionada, es decir, dar visibilidad a aquellos trabajos de investigación que tratan de explicar desde todas las perspectivas y enfoques posibles qué efectos tiene el contrato psicológico sobre el compromiso organizacional. Se han utilizado técnicas bibliométricas que aportan un horizonte detallado sobre la producción de estudios empíricos, permitiendo ver de forma conjunta el potencial de la relación entre ambos conceptos.

De los resultados de este trabajo, se desprende que, efectivamente, hay una importante producción que relaciona los conceptos contrato psicológico y compromiso organizacional. Esta comienza a principios de los años noventa del siglo pasado, de forma poco relevante, y no es hasta 2005 cuando realmente toma fuerza el interés por esta temática. Los años más fructíferos en cuanto al número de trabajos fueron entre 2006 y 2015, donde se concentró el 72\% de toda la muestra. Hay que indicar que los trabajos publicados tanto en WOS como en SCOPUS lo hacían en revistas que en su mayoría pertenecían al primer y segundo cuartil de dichas bases de datos, con lo cual le imprimen a la temática un cierto nivel de relevancia; sin embargo, ninguna de las revistas representa más del 10\% de artículos publicados, lo que evidencia que no existe especialización del tema por ninguna de ellas. En cuanto a la producción por países, Estados Unidos e Inglaterra han publicado más del $42 \%$ de los trabajos, en el caso de España los trabajos publicados han sido siete.

En cuanto a los enfoques o perspectivas que han estudiado contrato psicológico y compromiso organizacional, en la revisión se han encontrado trabajos 
que abordan ambos conceptos como eje principal, sin embargo, hay otros que lo han hecho de forma separada y utilizando moderadores tales como la tipología de empleo, moderadores demográficos y análisis generacionales, entre otros. Con respecto a la evolución y el nivel de producción, aquellas investigaciones relacionadas con aspectos organizacionales han tenido un total de 63 publicaciones $(30,4 \%)$. Los temas principales han sido la inseguridad laboral, el papel del supervisor en el compromiso organizacional o la justicia o ética organizacional. Otro de los enfoques más prolíferos ha sido la gestión de RR. HH., con un total de 32 artículos $(15,5 \%)$, con investigaciones relacionadas con la carrera profesional o la externalización de los RR. HH. Entre los enfoques de investigación con una producción menor, en cinco artículos $(2,4 \%)$ se encuentra el orientado al estudio de los factores motivacionales, con temas como los sentimientos hacia la organización y factores de contención. Por otra parte, se ha podido comprobar que temas tan sensibles como la gestión de la diversidad, la repercusión en la igualdad de género o aspectos sobre la cultura organizacional están escasamente investigados. En cuanto a los estudios relacionados con gestión de la diversidad, con trabajos sobre etnia y raza, interculturalidad o multiculturalidad, solo se han encontrado siete publicaciones $(3,3 \%)$ : tres investigan etnia y raza y cuatro lo hacen sobre multiculturalidad e interculturalidad. En el caso de las primeras, todas han sido publicadas por los mismos autores. En cuanto a la producción relacionada con género, es exigua, solo hay cinco trabajos que lo utilizan como moderador. Por último, sobre cultura organizacional, dos trabajos han profundizado en este tema y lo han relacionado con la satisfacción y el compromiso organizacional, y su eficacia como mecanismo de control en el contexto multinacional.

Para futuras investigaciones relacionadas con compromiso organizacional y contrato psicológico se propone explorar y profundizar en ambos conceptos en relación con temas menos investigados, tales como gestión de la diversidad, igualdad de género y cultura organizacional. 


\section{Referencias bibliográficas}

Ainsworth, S. y Purss, A. (2009). "Same time, next year? Human resource management and seasonal workers". Personnel Review, 38 (3), 217-235. DOI: 10.1108/00483480910943304.

Argrris, C. (1960)."Understanding organizational behavior” (p.xii+179). Dorsey Press. Recuperado de: <http://hdl.handle.net/2027/mdp.39015001648545>. Arnold, J. (1996). "The psychological contract: A concept in need of closer scrutiny?" European Journal of Work and Organizational Psychology, 15, 511520.

Becker, H. S. (1960). "Notes on the concept of commitment". American Journal of Sociology, 66, 350-360.

Bellou, V. (2009). "Profiling the desirable psychological contract for different groups of employees: evidence from Greece". The International Journal of Human Resource Management, 20 (4), 810-830. Disponible en: <https://doi. org/10.1080/09585190902770711>.

Benavides, C.; Guzmán, V. y Quintana, C. (2011). "Evolución de la literatura sobre empresa familiar como disciplina científica". Cuadernos de economía y dirección de la empresa, 14 (2), 78-90. Disponible en: <https://doi. org/10.1016/j.cede.2011.02.004>.

Betanzos, N. y Paz, F. (2007). "Análisis psicométrico del compromiso organizacional como variable actitudinal". Anales de Psicología, 23 (2), 207-215.

Buchanan, B. (1974). "Building Organizational Commitment: The Socialization of Managers in Work Organizations". Administrative Science Quarterly, 19 (4), 533-546.

Buttner, E.; Lowe, K. y Billings-Harris, L. (2010). "The Impact of Diversity Promise Fulfillment on Professionals of Color Outcomes in the USA". Journal of Business Ethics, 91 (4), 501-518. Disponible en: <https:// doi.org/10.1007/s10551-009-0096-y>.

Buttner, E.; Lowe, K. y Billings-Harris, L. (2012). "An Empirical Test of Diversity Climate Dimensionality and Relative Effects on Employee of Color Outcomes". Journal of Business Ethics, 110 (3), 247-258. Disponible en: $<$ https://doi.org/10.1007/s10551-011-1179-0>.

Callon, M.; Courtial, J. y Penan, H. (1995). Cienciometría: la medición de la actividad científica, de la bibliometría a la vigilancia tecnológica. Gijón: Trea.

Cantisano, G.; Domínguez, J. y Depolo, M. (2008). “Psychological contract breach and outcomes: combining meta-analysis and structural equation models". Psicothema, 20 (3), 487-496. 
Carlson, D. S. y Perrewe, P. L. (1995). "Institutionalization of organizational ethics through transformational leadership." Journal of Business Ethics, 14 (10), 829-838. DOI: $10.1007 /$ bf00872349.

Cheng, G. y Chan, D. (2008). "Who Suffers More from Job Insecurity? A Meta-Analytic Review". Applied Psychology, 57(2), 272-303. Disponible en: <https://doi.org/10.1111/j.1464-0597.2007.00312.x>.

Conway, N.; Guest, D. y Trenberth, L. (2011). "Testing the differential effects of changes in psychological contract breach and fulfillment". Journal of Vocational Behavior, 79 (1), 267-276. DOI: 10.1016/j.jvb.2011.01.003.

Сотton, J. L. (1993). Employee involvement. New York: Sage.

Courtial, J. P. (1994).“A co-word analysis of scientometrics”. Scientometrics, 31, 251-260.

Coyle-Shapiro, J. A. M.; Morrow, P. C;; Richardson, R. y Dunn, S. R. (2002). "Using profit sharing to enhance employee attitudes: A longitudinal examination of the effects on trust and commitment". Human Resource Management, 41 (4), 423-439. DOI: 10.1002/hrm.10052.

Coyle-Shapiro, J. A. M. y Morrow, P. C. (2006). “Organizational and client commitment among contracted employees". Journal of Vocational Behavior, 68 (3), 416-431. DOI: 10.1016/j.jvb.2005.10.002.

Enache, M.; Sallán, J.; Simo, P. y Fernández, V. (2013). “Organizational commitment within a contemporary career context". International Journal of Manpower, 34 (8), 880-898.

Gelade, G.; Dobson, P. y Auer, K. (2008). "Individualism, Masculinity, and the Sources of Organizational Commitment". Journal of CrossCultural Psychology, 39 (5), 599-617. Disponible en: <https://doi. org/10,1177/0022022108321308>.

Georgellis, Y. y Lange, T. (2007). "Participation in continuous, on-thejob training and the impact on job satisfaction: longitudinal evidence from the German labour market". The International Journal of Human Resource Management, 18 (6), 969-985. Disponible: <https://doi. org/10.1080/09585190701321112>.

Gordon, G. y Levinson, H. (1964). "Men, Management, and Mental Health”. Industrial and Labor Relations Review, 17 (2).

Granrose, C. S. y Baccili, P. A. (2006). “Do psychological contracts include boundaryless or protean careers?" Career Development International, 11 (2), 163-182. DOI: $10.1108 / 13620430610651903$.

Gregory, D. M.; Way, C. Y.; LeFort, S.; Barrett, B. J., y Parfrey, P. S. (2007). "Predictors of registered nurses' organizational commitment and 
intent to stay". Health Care Management Review, 32 (2), 119-127. DOI: 10.1097/01.HMR.0000267788.79190.f4.

Guest, D. E. (1998). "Is the psychological contract worth taking seriously?" J. Organiz. Behav., 19, 649-664.

Guzzo, R. A.; Noonan, K. A. y Elron, E. (1994). Expatriate Managers and the Psychological Contract". Journal of Applied Psychology, 79 (4), 617-626. DOI: 10.1037/0021-9010.79.4.617.

Haggard, D. L+ (2012). "Mentoring and Psychological Contract Breach”. Journal of Business and Psychology, 27 (2), 161-175. DOI: 10.1007/s10869-0119237-2.

Holly Buttner, E*; Lowe, K. y Billings-Harris, L. (2010). "Diversity climate impact on employee of color outcomes: does justice matter?" Career Development International, 15 (3), 239-258. Disponible en: <https $\% / /$ doi. org/10.1108/13620431011053721>.

Keim, A.; Landis, R.; Pierce, C. y Earnest, D. (2014). "Why Do Employees Worry About Their Jobs? A Meta-Analytic Review of Predictors of Job Insecurity". Journal of Occupational Health Psychology, 19 (3), 269-290. Disponible en: <https;//doi.org/10.1037/a0036743>.

Kickul, J.; Lester, S. y Belgio, E. (2004). "Attitudinal and Behavioral Outcomes of Psychological Contract Breach: A Cross Cultural Comparison of the United States and Hong Kong Chinese". International Journal of Cross Cultural Management, 4 (2), 229-252. Disponible en: <https //doi. org/10.1177/1470595804044751>.

Lapalme, M. E.; Simard, G. y Tremblay, M. (2011). "The Influence of Psychological Contract Breach on 'Temporary Workers' Commitment and Behaviors: A Multiple Agency Perspective". Journal of Business and Psychology, 26 (3), 311-324. DOI: 10.1007/s10869-010-9190-5.

Magoshi, E. y Chang, E. (2009). "Diversity management and the effects on employees' organizational commitment: Evidence from Japan and Korea". Journal of World Business, 44 (1), 31-40. Disponible en: <https://doi. org/10.1016/j.jwb.2008.03.018>.

Mathieu, J. E. y Zajac, D. M. (1990). "A review and meta-analysis of the antecedents, correlates, and consequences of organizational commitment". Psychological Bulletin, 108, 171-194.

McDonald, D. J. y Makin, P. J. (2000). “The psychological contract, organisational commitment and job satisfaction of temporary staff". Leadership \& Organization Development Journal, 21 (2), 84-91. DOI: $10.1108 / 01437730010318174$. 
Millward, L. J. y Hopkins, L.J. (1998). "Psychological contracts, organizational and job commitment”. Journal of Applied Social Psychology, 28 (16), 15301556. DOI: 10.1111/j.1559-1816.1998.tb01689.x.

Mowday, R. T., Steers, R. M. y Porter, L. W+ (1979). "The measurement of organizational commitment". Journal of Vocational Behavior, 14, 224-247.

Ng, T. W. H. y Feldman, D. C. (2009)."Re-examining the relationship between age and voluntary turnover". Journal of Vocational Behavior, 74 (3), 283-294. DOI $10.1016 /$ j.jvb.2009.01.004.

O Reilly, C. A. y Chatman, J. (1986). "Organizational commitment and psichological attachment: The effects of compliance, identification and internalization on prosocial behavior". Journal of Applied Psychology, 71, 492 499.

Parzefall, M. R. y Hakanen, J. (2010). "Psychological contract and its motivational and health-enhancing properties". Journal of Managerial Psychology, 25 (1), 4-21. DOI: 10.1108/02683941011013949.

Porter, L. W. y Lawer, E. E. (1965). Managerial attitudes and performance. Irwin: Homewood.

Richard, O. C.; McMillan-Capehart, A.; Bhuian, S. N. y Taylor, E. C. (2009). "Antecedents and consequences of psychological contracts: Does organizational culture really matter?” Journal of Business Research, 62 (8), 818-825. DOI: 10.1016/j,jbusres.2008.04.001.

Rousseau, D. M. (1995). Psychological Contracts in Organisations. Understanding Written and Unwritten Agreements. USA: SAGE Publications.

Rousseau, D. M. y Parks, J. (1993). "The contracts of individuals and organizations". En L. L. Cummings y B. M. Staw (eds.). Research in Organizational Behavior, vol. 15 (pp. 1-43). Greenwich: JAI Press.

Scandura, T+ y Lankau, M. (1997). "Relationships of gender, family responsibility and flexible work hours to organizational commitment and job satisfaction". Journal of Organizational Behavior, 18 (4), 377-391. Disponible en: <https://doi.org/10.1002/(SICI)1099-1379(199707)18:43.0.CO;2-1>.

Schein, E. H. (1965). Organizational psychology. New Jersey: Prentice-Hall. Schein, E. H. (1982). Psicología de las Organizaciones. Madrid: Prentice-Hall.

SeOng, J., Hong, D. y PARK, W. (2012). "Work status, gender, and organizational commitment among Korean workers: The mediating role of personorganization fit". Asia Pacific Journal of Management, 29 (4), 1105-1129. Disponible en: <https://doi.org/10.1007/s10490-011-9248-6>. 
Silla, I.; Gracia, F; Ángel Mañas, M. y Peiró, J. (2010). "Job insecurity and employees' attitudes: the moderating role of fairness". International Journal of Manpower, 31 (4), 449-465.

Silverthorne, C. (2004). "The impact of organizational culture and personorganization fit on organizational commitment and job satisfaction in Taiwan”. Leadership E Organization Development Journal, 25 (7), 592-599. DOI: 10.1108/01437730410561477.

Simó, P; Enache, M.; Sallán, J. M. y Fernández, V. (2010). "Analysis of the Relation Between Subjective Career Success, Organizational Commitment and the Intention to Leave the Organization". Transylvanian Review of Administrative Sciences, 29E/2010, 144-158.

Sims, R. R. (1991). "The institutionalization of organizational ethics." Journal of Business Ethics, 10 (7), 493-506. DOI: 10.1007/BF00383348.

Singh, V. y Vinnicombe, S. (2000). "What does "commitment" really mean?: Views of UK and Swedish engineering managers". Personnel Review, 29 (2), 228-254. Disponible en: <https://doi.org/10.1108/00483480010296014>. Solinger, O. N.; Hofmans, J., Bal; P. M., y Jansen, P. G. W.(2016)."Bouncing back from psychological contract breach: How commitment recovers over time". Journal of Organizational Behavior, 37 (4), 494-514. doi:10.1002/ job.2047.

Sora, B.; Caballer, A.; Peiró, J. y De Witte, H. (2009). "Job insecurity climate's influence on employees' job attitudes: Evidence from two European countries". European Journal of Work and Organizational Psychology, 18(2), $125-147$.

Sora, B.; Caballer, A. y Peiró, J. (2010). "The consequences of job insecurity for employees: The moderator role of job dependence". International Labour Review, 149(1), 59-72.

Stoner, J. y Gallagher, V. (2010). "Who Cares? The Role of Job Involvement in Psychological Contract Violation". Journal of Applied Social Psychology, 40 (6), 1490-1514. Disponible en: <https://doi.org/10.1111 /j.1559-1816.2010.00626.x>.

Susaeta, L.; Piñeiro, J.; Idrovo Carlier, S.; Espejo, A. y Belizón, M. (2013). "Generation or culture? Work attitude drivers; an analysis in Latin America and Iberian countries". Cross Cultural Management : an International Journal, 20(3), 321-360. Disponible enः <https //doi.org/10.1108/CCM12-2011-0126>.

Tang, T.; Cunningham, P.; Frauman, E.; Ivy, M.y Perry, T. (2012)."Attitudes and Occupational Commitment among Public Personnel: Differences 
between Baby Boomers and Gen-Xers". Public Personnel Management, 41 (2), 327-360. Disponible en: <https://doi.org/10.1177/00910260120410020>. TenA, G. (2002)."El contrato psicológico: Relación laboral empresa-trabajador". Acciones e Investigaciones Sociales, 15, 85-107.

Tomprou, M.; Rousseau, D. M. y Hansen, S. D. (2015)."The psychological contracts of violation victims: A post-violation model". Journal of Organizational Behavior, 36 (4), 561-581. DOI: 10.1002/job.1997.

Topa Cantisano, G. y Palací Descals, F. (2004). “¿Ruptura o cumplimiento del contrato psicológico?: una revisión meta-analítica de la investigación empírica". Acción psicológica, 3 (3), 155-177. Disponible en: <https://doi. org/10.5944/ap.3.3.510>.

Welch, D.y Welch, L. (2006).“Commitment for hire? The viability of corporate culture as a MNC control mechanism”. International Business Review, 15 (1), 14-28. Disponible en: <https://doi.org/10.1016/j.ibusrev.2005.10.001>.

Willem, A.; De Vos, A., y Buelens, M. (2010). “Comparing Private and Public Sector Employees' Psychological Contracts: Do they attach equal importance to generic work aspects?" Public Management Review, 12 (2), 275-302. Disponible en. <https://doi.org/10.1080/14719031003620323>.

Yeh, Y. J. Y.; Ko, J. J. R.; Chang, Y. S. y Chen, C. H. V. (2007). "Job stress and work attitudes between temporary and permanently employed nurses." Stress and Health, 23 (2), 111-120. DOI: 10.1002/smi.1128.

Zhao, H.; Wayne, S.; Glibkowski, B. y Bravo, J. (2007). "The impact of psychological contract breach on work-related outcomes: a meta-analysis". Personnel Psychology, 60 (3), 647-680.

\section{Recursos electrónicos}

Bases de datos WOS y Scopus disponibles en la Fundación Española para la Ciencia y la Tecnología). FEYCT [Consulta: 31 marzo 2019]. Disponible en $<$ https://www.recursoscientificos.fecyt.es/ > . 


\section{Anexos}

\section{Anexo I. Enfoques temáticos}

\begin{tabular}{|c|c|c|c|c|c|c|c|}
\hline Aspectos organizacionales & 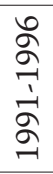 & 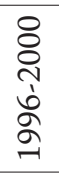 & 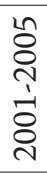 & 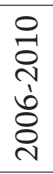 & 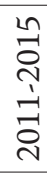 & 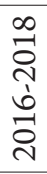 & 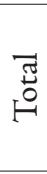 \\
\hline Inseguridad laboral & & & 2 & 8 & 4 & 2 & 16 \\
\hline $\begin{array}{l}\text { Papel del supervisor en el compromiso } \\
\text { organizacional }\end{array}$ & & & & 6 & 4 & 1 & 11 \\
\hline Justicia organizacional & & & 1 & 3 & 3 & 1 & 8 \\
\hline Ética organizacional & 2 & & & & 5 & & 7 \\
\hline Cambios en la organización & & 1 & & 2 & 3 & & 6 \\
\hline Responsabilidad corporativa & & & & & 3 & & 3 \\
\hline Conciliación familiar & & 1 & 1 & & & & 2 \\
\hline Cultura organizacional & & & 1 & 1 & & & 2 \\
\hline Apoyo organizacional & & & 1 & & 1 & & 2 \\
\hline Agotamiento en el trabajo & & 1 & & & 1 & & 2 \\
\hline Comunidad & & 1 & & & & & 1 \\
\hline Plan de acogida & & & & & 1 & & 1 \\
\hline Clima laboral & & & & 1 & & & 1 \\
\hline Conducta organización ciudadanía & & & & 1 & & & 1 \\
\hline Total artículos & 2 & 4 & 6 & 22 & 25 & 4 & 63 \\
\hline
\end{tabular}

Fuente: elaboración propia a partir de datos de WOS y Scopus.

\begin{tabular}{|c|c|c|c|c|c|c|c|}
\hline Moderadores & 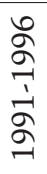 & 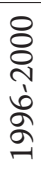 & 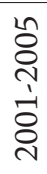 & 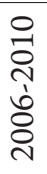 & 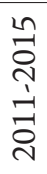 & 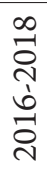 & تేّ \\
\hline Tipología de empleo & & & 3 & 4 & 2 & & 9 \\
\hline Moderadores demográficos & & & & 2 & 4 & & 6 \\
\hline Expatriados/impatriados & 1 & 1 & & 3 & & & 5 \\
\hline Factores personales & & 1 & 2 & 1 & 1 & & 5 \\
\hline Análisis generacional & & & & 1 & 2 & 1 & 4 \\
\hline Condicionamiento sindical & & 1 & 1 & & & & 2 \\
\hline $\begin{array}{l}\text { Trabajo profesional } v s, \text { trabajo } \\
\text { administrativo }\end{array}$ & & & 1 & & & & 1 \\
\hline
\end{tabular}




\begin{tabular}{|l|r|r|r|r|r|r|r|}
\hline Contrato normativo & & & & & 1 & & 1 \\
\hline Imagen de la empresa & & & & & & 1 & 1 \\
\hline Sector privado vs. sector público & & & & 1 & & & 1 \\
\hline Total artículos & 1 & 3 & 7 & 12 & 10 & 2 & 35 \\
\hline
\end{tabular}

Fuente: elaboración propia a partir de datos de WOS y Scopus.

\begin{tabular}{|c|c|c|c|c|c|c|c|}
\hline Gestión RR. HH. & $\begin{array}{l}\frac{\curvearrowright}{\sigma} \\
\frac{1}{\sigma} \\
\text { }\end{array}$ & $\begin{array}{l}8 \\
8 \\
\text { ஸे } \\
\text { ঠे } \\
\text { - }\end{array}$ & 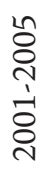 & $\begin{array}{l}\text { 을 } \\
\text { ㄱ. } \\
\text { பे } \\
\text { ठ্ণ }\end{array}$ & 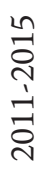 & 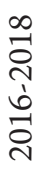 & 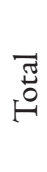 \\
\hline Carrera profesional & & 2 & 1 & 2 & 3 & & 8 \\
\hline Externalización de RR. HH. & & & & 2 & 2 & & 4 \\
\hline Formación & & & & 2 & 1 & 1 & 4 \\
\hline Innovación en RR. HH. & & & 1 & 2 & & & 3 \\
\hline Prácticas de RR. HH. & & 2 & & & 1 & & 3 \\
\hline Enfoques estratégicos de RR. HH. & & & 1 & 2 & & & 3 \\
\hline Aprendizaje y retención del talento & & & & 1 & 1 & & 2 \\
\hline $\begin{array}{l}\text { Inversión capital humano vs. } \\
\text { externalización RR. HH. }\end{array}$ & & 1 & & & 1 & & 2 \\
\hline Sistema de compensación & & & & 1 & & & 1 \\
\hline Aspectos cognitivos & & & & & 1 & & 1 \\
\hline Gestión del conocimiento & & & 1 & & & & 1 \\
\hline Total artículos & 0 & 5 & 4 & 12 & 10 & 1 & 32 \\
\hline
\end{tabular}

Fuente: elaboración propia a partir de datos de WOS y Scopus.

\begin{tabular}{|c|c|c|c|c|c|c|c|}
\hline $\begin{array}{l}\text { Contrato psicológico - Compromiso } \\
\text { organizacional }\end{array}$ & $\begin{array}{l}\stackrel{2}{2} \\
\stackrel{2}{-} \\
\stackrel{-}{2}\end{array}$ & $\begin{array}{l}8 \\
\text { ठ } \\
\text { ஸे } \\
\text { ু } \\
\text { - }\end{array}$ & 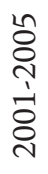 & $\begin{array}{l}0 \\
\text { o } \\
\text { సे } \\
\text { ஸे } \\
\text { ठ }\end{array}$ & 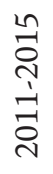 & 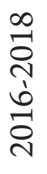 & 퓸 \\
\hline $\begin{array}{l}\text { Contrato psicológico - compromiso } \\
\text { organizacional }\end{array}$ & 0 & 0 & 2 & 7 & 12 & 1 & 22 \\
\hline \multicolumn{8}{|l|}{ Contrato psicológico } \\
\hline Desenlace del contrato psicológico & & & & 7 & 6 & 1 & 14 \\
\hline $\mathrm{CP}$ relacional vs, transaccional & & 2 & & 5 & 1 & & 8 \\
\hline
\end{tabular}




\begin{tabular}{|c|c|c|c|c|c|c|c|}
\hline $\begin{array}{l}\text { Factores que influyen en el contrato } \\
\text { psicológico }\end{array}$ & & & & & & 1 & 1 \\
\hline \multirow[t]{2}{*}{ Análisis metodológico } & & & & 1 & & & 1 \\
\hline & 0 & 2 & 0 & 13 & 7 & 2 & 24 \\
\hline \multicolumn{8}{|l|}{ Compromiso organizacional } \\
\hline Rotación & & & 5 & 2 & & 1 & 8 \\
\hline $\begin{array}{l}\text { Factores que influyen en el compromiso } \\
\text { organizacional }\end{array}$ & & & 1 & 1 & 1 & 1 & 4 \\
\hline Dimensiones del compromiso & & & & 1 & 2 & & 3 \\
\hline Rotación voluntaria & & & & 1 & 1 & & 2 \\
\hline \multirow[t]{2}{*}{ Ejecutivos y compromiso organizacional } & & 1 & & & 1 & & 2 \\
\hline & 0 & 1 & 6 & 5 & 5 & 2 & 19 \\
\hline Total artículos & 0 & 3 & 8 & 25 & 24 & 5 & 65 \\
\hline
\end{tabular}

Fuente: elaboración propia a partir de datos de WOS y Scopus.

\begin{tabular}{|c|c|c|c|c|c|c|c|}
\hline Diversidad & $\begin{array}{l}\frac{\curvearrowright}{\sigma} \\
\frac{1}{2} \\
\text { ন }\end{array}$ & $\begin{array}{l}8 \\
\text { ํ. } \\
\text { ஸे } \\
\text { ু }\end{array}$ & 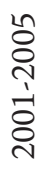 & 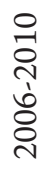 & 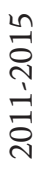 & 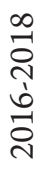 & $\stackrel{\text { تే }}{\circ}$ \\
\hline Etnia y raza & & & & 3 & 1 & & 4 \\
\hline Interculturalidad & & & 1 & & 1 & & 2 \\
\hline Multiculturalidad & & & & 1 & & & 1 \\
\hline Total artículos & 0 & 0 & 1 & 4 & 2 & 0 & 7 \\
\hline
\end{tabular}

Fuente: elaboración propia a partir de datos de WOS y Scopus.

\begin{tabular}{|c|c|c|c|c|c|c|c|}
\hline Factores motivacionales & $\begin{array}{l}\circ \\
\stackrel{2}{-} \\
\frac{1}{2} \\
\frac{\sigma}{-1}\end{array}$ & 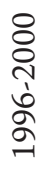 & 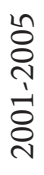 & 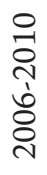 & 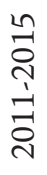 & 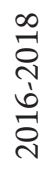 & 茕 \\
\hline Factores motivacionales intrínsecos & & & 1 & & 1 & & 2 \\
\hline Sentimientos hacia la organización & & & & & 2 & & 2 \\
\hline Factores contención & & & & 1 & & & 1 \\
\hline Total artículos & 0 & 0 & 1 & 1 & 3 & 0 & 5 \\
\hline
\end{tabular}

Fuente: elaboración propia a partir de datos de WOS y Scopus. 